\title{
Design and Evaluation of a 3-D Printed Optical Sensor for Monitoring Finger Flexion
}

\author{
Lefan Wang, Turgut Meydan, Member, IEEE, and Paul Williams
}

\begin{abstract}
The development of techniques for monitoring finger movement is becoming increasingly important in areas, such as robotics, virtual reality, and rehabilitation. To date, various techniques have been proposed for tracking hand movements, but the majority suffer from poor accuracy and repeatability. Inspired by the articulated structure of finger joints, we propose a novel 3-D printed optical sensor with a compact hinged configuration for tracking finger flexion. This sensor exploits Malus' law using the attenuation of light transmitted through crossed polarizers. The sensor consists of a single LED, two pieces of linear polarizing film, and a photodetector that detects the changes in polarized light intensity proportional to the angle of finger flexion. This paper presents the characterization of the proposed optical sensor and compares it with a commonly used commercial bend sensor. Results show that the bend sensor exhibits hysteresis error, low sensitivity at small angles, and significant temporal drift. In contrast, the optical sensor is more accurate $\left( \pm 0.5^{\circ}\right)$ in the measuring range from $0^{\circ}$ to $90^{\circ}$, and exhibits high repeatability and stability, as well as a fast dynamic response. Overall, the optical sensor outperforms the commercial bend sensor, and shows excellent potential for monitoring hand movements in real time.
\end{abstract}

Index Terms-Angle measurement, bend sensor, glove-based system, hinged configuration, hand motion tracking, optical sensor, 3-D printing.

\section{INTRODUCTION}

$\mathbf{M}$ ONITORING hand movement is an important requirement in areas such as robotics, physical rehabilitation and therapy, virtual reality, and sign language recognition, to name a few examples [1]. Since the 1970s, considerable attention has been focused on researching methods for tracking hand movement [1], [2]. The complexity of hand articulation (up to 27 degrees of freedom) combined with a lack of suitable sensing devices has limited the development of accurate, low profile solutions for monitoring hand posture and motion.

Current hand tracking devices can be categorized into two main types: camera-based systems and glove-based systems. Camera systems detect either the hand contour [2] or small markers including retro-reflective spheres [3] and LEDs [4] attached to the finger segments. The major limitation of such systems is that the measurement can only be performed in a restricted range determined by the position of the cameras.

Manuscript received July 11, 2016; revised January 13, 2017; accepted January 14, 2017. Date of publication January 19, 2017; date of current version February 17, 2017. This work was supported in part by Cardiff University and in part by China Scholarship Council. The associate editor coordinating the review of this paper and approving it for publication was Dr. Anna G. Mignani.

The authors are with the Wolfson Centre for Magnetics, School of Engineering, Cardiff University, Cardiff CF24 3AA, U.K. (e-mail: meydan@cardiff.ac.uk).

Digital Object Identifier 10.1109/JSEN.2017.2654863

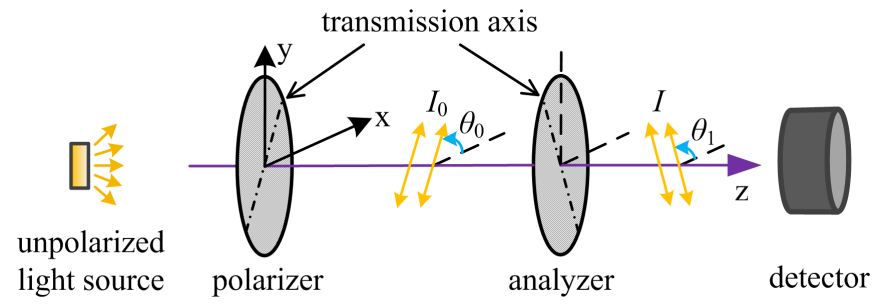

Fig. 1. The schematic diagram of the optical sensor. Both the polarizer and the analyzer are two pieces of linear polarizing film, and the photodetector is used to measure the final light intensity.

Additionally, the self-occlusion of the subjects' hands or poor image quality can lead to inaccuracies. By contrast, the glove-based systems measure angular positions directly and are unaffected by finger occlusion. Subsequently, glove-based systems have become the dominant devices for capturing hand motion.

Various sensing elements have been proposed for use in instrumented gloves including resistance sensors, magnetic sensors, and fibre-optic sensors. Resistance sensors include flexible wires [5], conductive polymer PEDOT: PSS [6], graphene woven fabrics [7], silver nanomaterials [8], liquidembedded elastomer electronics [9], and stretchable carbon nanotubes [10]. Although the resistance sensors are typically lightweight, flexible, low cost, and suitable for wearable devices, their performance often suffers from signal drift and instability. In general, this problem can be overcome by adopting a time-consuming calibration procedure [9], [11].

For magnetic techniques, permanent magnets are used as reference points and the position of the hands with respect to the magnetic sources are detected by magnetic sensors [12]-[15]. The magnetic sensors are capable of providing precise hand positioning data, but they are prone to inaccuracies due to interference from the Earth's geomagnetic field or nearby ferromagnetic objects. They also rely on complicated models to convert the readings to relevant angular information [12], [15].

Fibre-optic sensors measure the bending angle (flexion) by detecting the attenuation of light passing through the fibre. To improve the sensitivity, optic fibres are made with imperfections [16] or polished [17]. Various types of sensors exist including hetero-core sensors consisting of single mode fibres with two different diameters [18], Fibre Bragg Grating (FBG) sensors [19], and flexible transducers with silicone rods [20]. These have all been used for tracking human body motion. Compared with resistance and magnetic sensors, fibre-optic sensors are reported to have higher stability and immunity to 


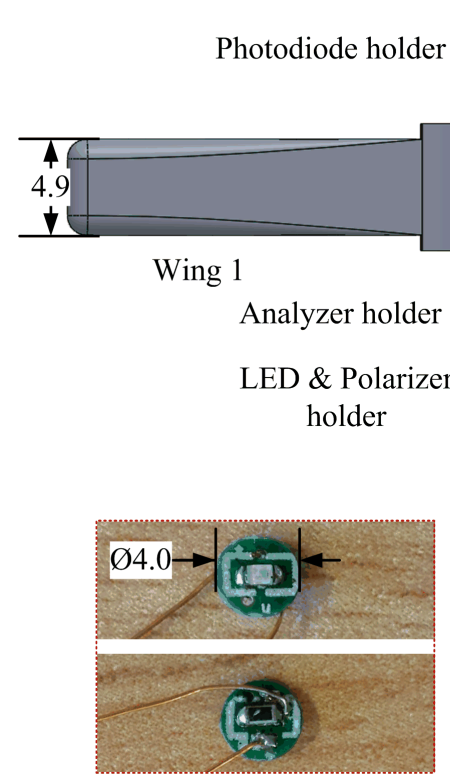

(c)

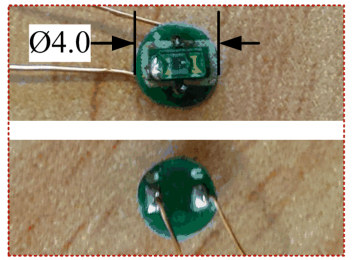

(d)

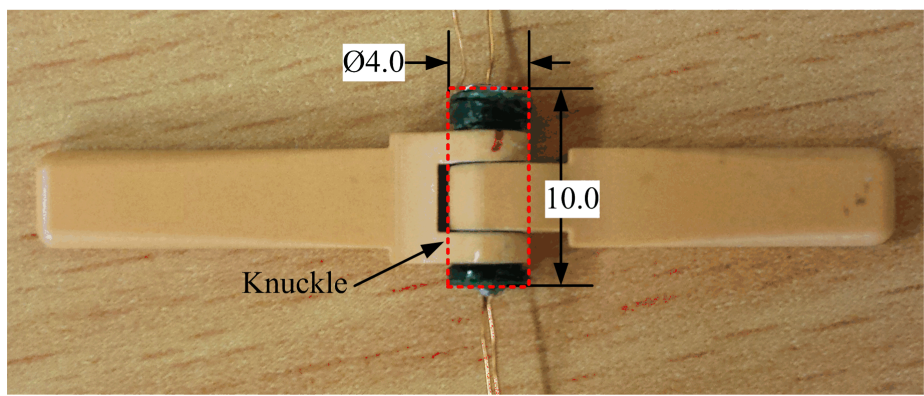

(e)

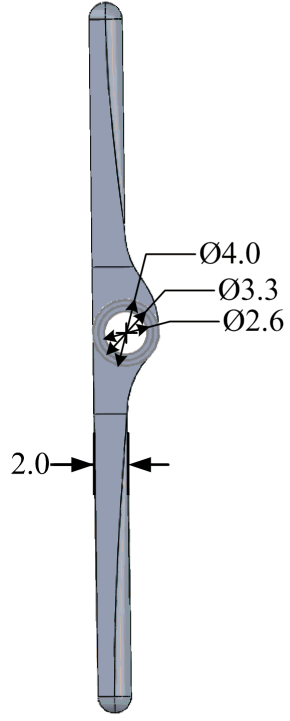

(b)

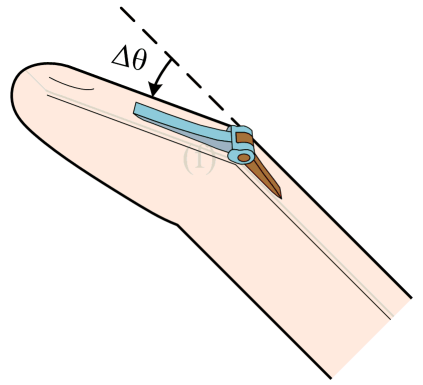

(f)

Fig. 2. The images of the 3D model and the complete optical sensor. All of the units are in millimetres: (a) and (b) are the front and lateral view of the 3D model, respectively; (c) and (d) are double-layered PCB boards for the LED, resistor (82.5 $\Omega$ ), and photodiode; (e) is the integrated optical sensor; and (f) a conceptual application of the optical sensor attached to the finger.

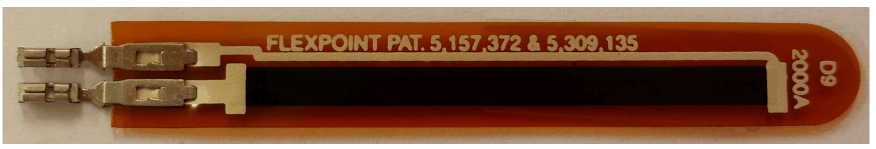

Fig. 3. The image of the commercial bend sensor.

electromagnetic interference [18], [20], [21]. A disadvantage is their lack of mobility due to the use of peripherals such as an optical power meter [18] or a CCD [20]. Lengthy calibration [18]-[20] procedures and, in the case of FBG, complex demodulation techniques limit the practicality of fibre-optic systems [19], [22]. For a user-friendly hand monitoring system, the sensing elements should meet the following criteria: high accuracy, low cost, compact and lightweight structure, and minimal calibration.

To satisfy the above criteria we have proposed and evaluated a novel optical sensor based on the principle of crossedpolarization detection. To the best of our knowledge, such a sensor is the first attempt to be used for monitoring human kinematics. The proposed sensor detects changes in polarized light intensity proportional to the bending angle seen in finger flexion. The sensor has a hinged configuration and is located directly above the finger joint such that it rotates

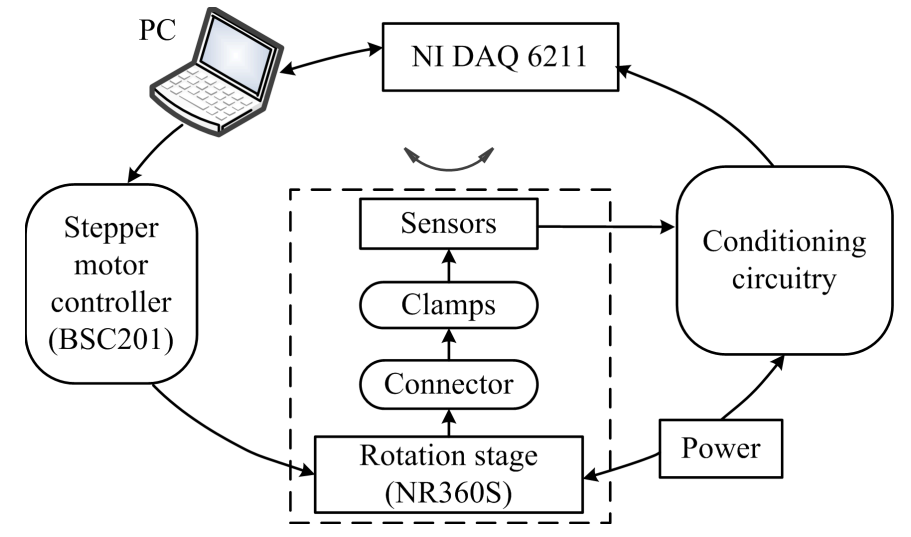

Fig. 4. The block diagram of the automated experimental set-up. Under the drive of the controller BSC201, the motorized stage NR360S is used to adjust the rotation speeds and angles. The optical and bend sensors are connected to the motor by some clamps. The sensor outputs are conditioned, sampled, and stored in the PC.

synchronously with the joint. In principle, the output will be independent of the radius of curvature of the joint. This eliminates the influence of joint size unlike the case for conventional flexible sensors. In this paper, we present the characteristics of our optical sensor and compare its performance with a 


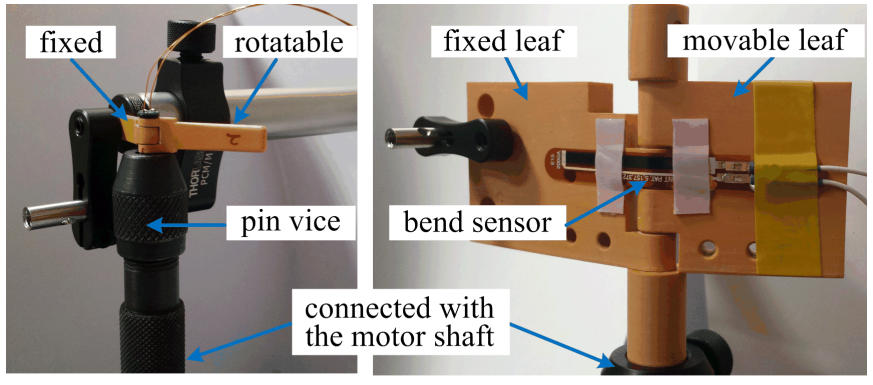

(a)

(b)

Fig. 5. Photograph of the mechanical set-up to hold (a) the optical sensor and (b) the bend sensor.

commercial bend sensor (Flexpoint Inc. [23]) which is widely used to track finger motion [24]-[26].

\section{THE PRINCIPLE OF OPERATION}

The optical sensor consists of a single LED light source, a linear polarizer, an analyzer, and a photodetector. The schematic diagram is shown in Fig. 1. One surface mounted LED (LTST-C193TGKT-5A, RS Components Ltd.), with a peak wavelength of $535 \mathrm{~nm}$, is employed as the light source. The incident light is initially polarized along the transmission axis of the polarizer, and then partly blocked by the analyzer depending on the analyzer's orientation relative to the polarizer. Both the polarizer and the analyzer are made from commercial linear polarizing sheet (Edmund Optics Ltd), with an extinction ratio of 9000:1 and high transmission from $400 \mathrm{~nm}$ to $700 \mathrm{~nm}$. The resultant light intensity is finally detected by a sensitive PIN photodiode (TEMD6200FX01, Farnell element14, UK) with a peak sensitivity at $540 \mathrm{~nm}$.

A three-dimensional coordinate system is defined as shown in Fig. 1, where the $\mathrm{z}$-axis is perpendicular to the plane of the polarizing film. Crossing the polarizer, $I_{0}$ is the initial polarized light intensity at $\theta_{0}$ degrees from the x-axis. Supposing the polarizing film is perfect, the intensity of polarized light passing through the analyzer, $I$, is given by (1) according to Malus' Law, where $\theta_{i}$ denotes the angle between the direction of the light's initial polarization and the analyzer's transmission axis, i.e. the difference between $\theta_{0}$ and $\theta_{1}$.

$$
I=I_{0} \cos ^{2} \theta_{i}=I_{0} \cos ^{2}\left(\theta_{1}-\theta_{0}\right)
$$

Therefore, the final light intensity for the optical sensor is proportional to the intersection angle of the transmission axes of the polarizer and the analyzer. The photodiode linearly converts the light intensity into an electric current $i$ (shown as (2)), where $k$ and $a$ are constants depending on the performance of the photodiode and polarizing sheet, and $m$ is the product of $k$ and $I_{0}$.

$$
i=k I+a=k I_{0} \cos ^{2} \theta_{i}+a=m \cos ^{2} \theta_{i}+a
$$

According to (2), angular rotation can be obtained by measuring the current $i$.

\section{The SEnsor Construction}

\section{A. Manufacture of the Optical Sensor}

The first prototype of the optical sensor was described in [27]. Here, we go on to describe miniaturization of the device together with additional performance characterization.

The front and lateral view of the sensor structure is shown in Fig. 2 (a) and (b), respectively. The edges require additional smoothing (post fabrication) to reduce sources of friction. The prototype is manufactured by EnvisionTEC's Perfactory Mini 3D Printer. The model is fabricated from Nanoparticlefilled material RCP30 due to its outstanding features including superior stiffness, opaque appearance, and its suitability for building parts at very high resolutions. After manufacture, the small parts including the holders for the LED, polarizer, analyzer, and photodiode are dyed black to increase opaqueness and reduce the effects of ambient light intrusion. The holders for the LED, polarizer, and photodiode are fixed to Wing 1 and the analyzer is housed in another holder placed inside Wing 2 . A rotation between Wing 1 and Wing 2 leads to the same rotation between the polarizer and analyzer.

The LED and photodetector are soldered onto doublelayered circular PCB boards with a thickness of $0.4 \mathrm{~mm}$ and a diameter of $4 \mathrm{~mm}$. Fig. 2 (c) and (d) show both sides of the LED board and photodiode board respectively.

The image of the complete optical sensor is shown in Fig. 2 (e). The outer diameter of the knuckle section is around $4 \mathrm{~mm}$ and the width approximately $10 \mathrm{~mm}$, which is suitable for most adults' fingers. Fig. 2 (f) illustrates the optical sensor on the dorsal surface of the finger. The method of the sensor's attachment to the finger or glove will be explored in the future. Based on our design, the sensor wings slide along the glove's surface to replicate the joint's flexion angle when the finger bends.

\section{B. The Sensor for Comparison}

Commercial flexible ink sensors from Flexpoint Inc. have been widely utilized in glove-based systems for hand movement detection due to their small weight, low cost, and flexibility. We evaluate the performance of a bend sensor, without an overlamination film, as a reference point for comparison with our sensor. The dimension of the bend sensor is $50.80 \mathrm{~mm} \times 7.00 \mathrm{~mm} \times 0.16 \mathrm{~mm}$ as seen in Fig. 3. This bend sensor consists of very thin and flexible material coated with a carbon/polymer based ink. When the sensor is bent, the ink develops micro cracks resulting in a change of resistance. The manufacturer's design guide indicates a dependence of the sensor's resistance on the radius of the curvature, and this has been experimentally verified elsewhere [28].

\section{EXPERIMENTAL Methods}

\section{A. Measurement Set-Up}

To reduce human operating errors and improve measurement consistency, an automated experimental set-up is built to assess the performances of both the optical and the bend sensor, see Fig. 4. A $360^{\circ}$ continuous motorized rotation stage (NR360S from Thorlabs Inc. [29]), with one arcsec resolution, 


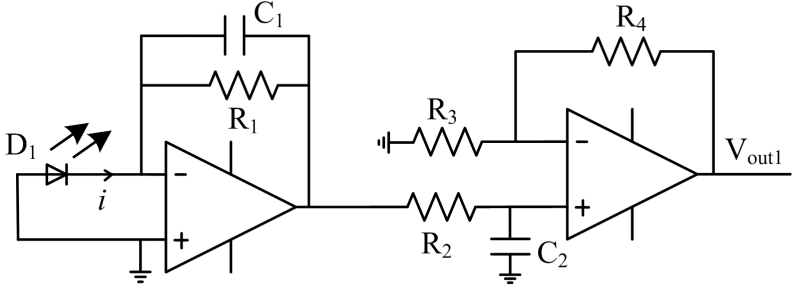

(a)

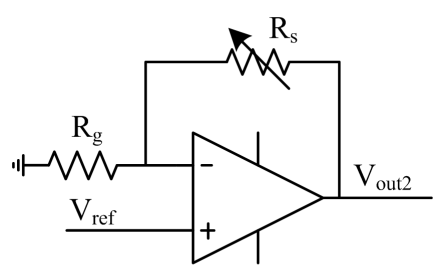

(b)

Fig. 6. The conditioning circuits. (a) The current-to-voltage converter for the optical sensor, $R_{1}=R_{2}=3 \mathrm{M} \Omega, \mathrm{R}_{3}=820 \Omega, \mathrm{R}_{4}=10 \mathrm{~K} \Omega$, $\mathrm{C}_{1}=3.3 \mathrm{pF}$, $\mathrm{C}_{2}=0.1 \mu \mathrm{F}$. (b) The resistance-to-voltage converter for the bend sensor, $\mathrm{V}_{r e f}=0.5 \mathrm{~V}, \mathrm{R}_{g}=39 \mathrm{~K} \Omega$, and $\mathrm{R}_{s}$ is the sensor resistance.

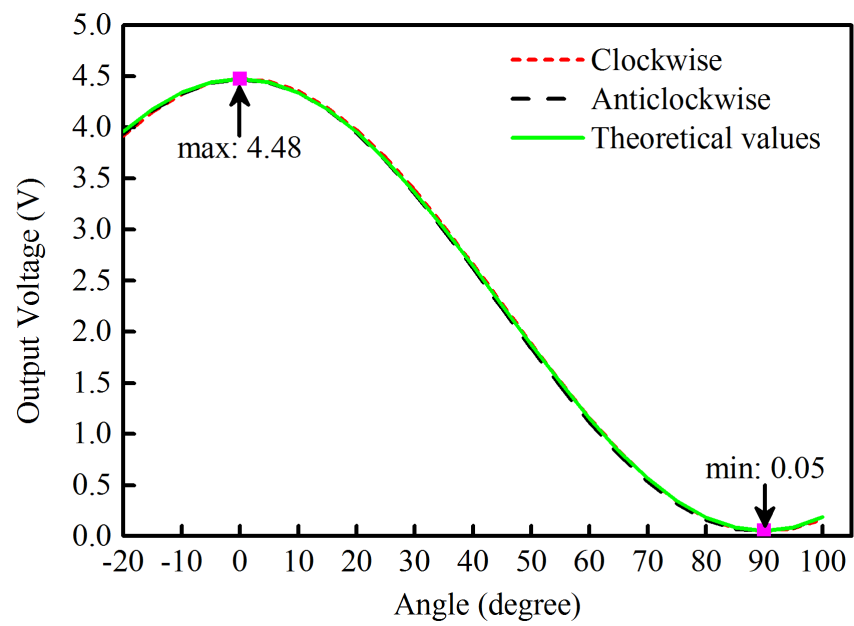

Fig. 7. Performance of the designed optical sensor in bidirectional motion ranged from $-20^{\circ}$ to $100^{\circ}$. The theoretical values are calculated according to Malus' law.

is employed to adjust the rotation angles and speeds. The accuracy of the motor is up to 5 arcmin and the maximum speed can be $50 \%$ when driven by the micro-stepping motor controller (BSC201). This controller offers 409600 microsteps per revolution, i.e. approximately $0.00088^{\circ}$ per step. The system components are controlled using ActiveX interfacing technology in LabVIEW together with signal acquisition using a National Instruments data acquisition card (NI USB-6211).

As is shown in Fig. 5 (a), a pin vice connected with the stepper motor is employed to hold the optical sensor. One of the sensor's wings is clamped, whilst the other wing is allowed to rotate under the guidance of the motorized stage. The bend sensor is fixed according to the method described by Saggio [6]. It is inserted into a plastic sleeve attached to the surface of a plastic hinge as shown in Fig. 5 (b). One leaf of the hinge is fixed whereas the other is rotated by the motor. The radius of curvature for this bend sensor is $8.5 \mathrm{~mm}$ during all of the tests in this paper.

\section{B. Conditioning Circuits}

The light intensity passing through the optical sensor varies with the angular rotation whereas the bend sensor exhibits changes in electrical resistance. The conditioning circuits for each sensor are shown in Fig. 6.

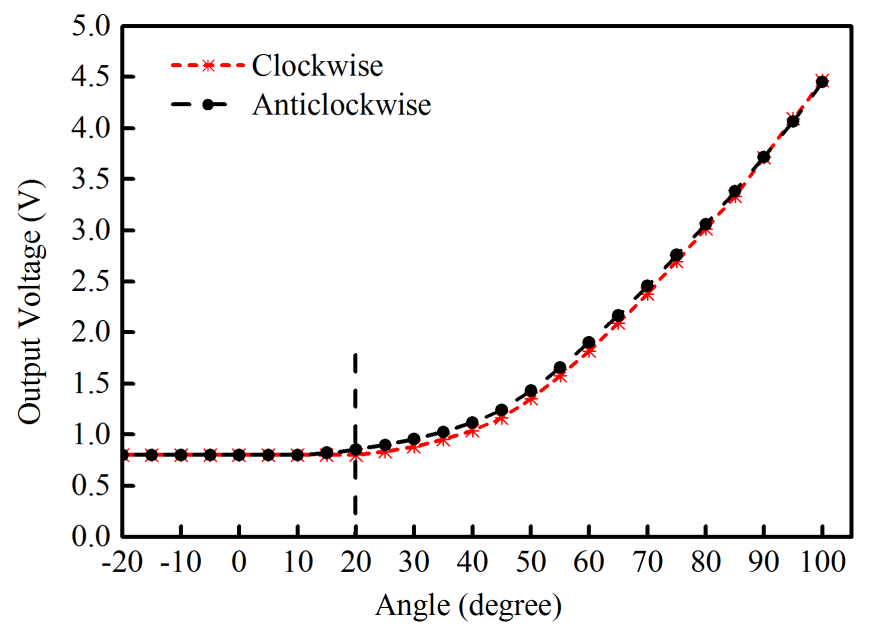

Fig. 8. Voltage-to-angle relationship of the bend sensor in bidirectional motion ranged from $-20^{\circ}$ to $100^{\circ}$.

In Fig. 6 (a), the photodiode (D1), which works in photovoltaic mode eliminating the possibility of dark current, linearly converts the received light intensity to electric current $i$. The parallel combination of $\mathrm{R}_{1}$ and feedback capacitor $\mathrm{C}_{1}$ determines the frequency response of the circuit. The signal is initially amplified by the feedback resistor $R_{1}$, and then modulated further by a second-stage amplifier with a low-pass filter. Finally, the output voltage can be obtained by (3).

$$
V_{\text {out } 1}=\frac{i R_{1}}{1+s C_{1} R_{1}}\left(1+\frac{R_{4}}{R_{3}}\right) \approx i R_{1}\left(1+\frac{R_{4}}{R_{3}}\right)
$$

Now substitute for the value of $i$ with (2),

$$
\begin{aligned}
V_{\text {out } 1} & =\left(m \cos ^{2} \theta_{i}+a\right) R_{1}\left(1+\frac{R_{4}}{R_{3}}\right) \\
& =n \cos ^{2} \theta_{i}+b
\end{aligned}
$$

where $n$ and $b$ are constants determined by the amplification factor and the parameters $m$ and $a$.

A non-inverting operational amplifier circuit (Fig. 6 (b)) is used for the bend sensor. $\mathrm{R}_{S}$ represents the sensor resistance, and $\mathrm{R}_{g}$ is a constant resistor and limits the output range. $\mathrm{V}_{\text {ref }}$ is a reference voltage applied to the positive input. Equation (5) is used to calculate the output voltage.

$$
V_{\text {out } 2}=V_{\text {ref }}\left(1+\frac{R_{S}}{R_{g}}\right)
$$




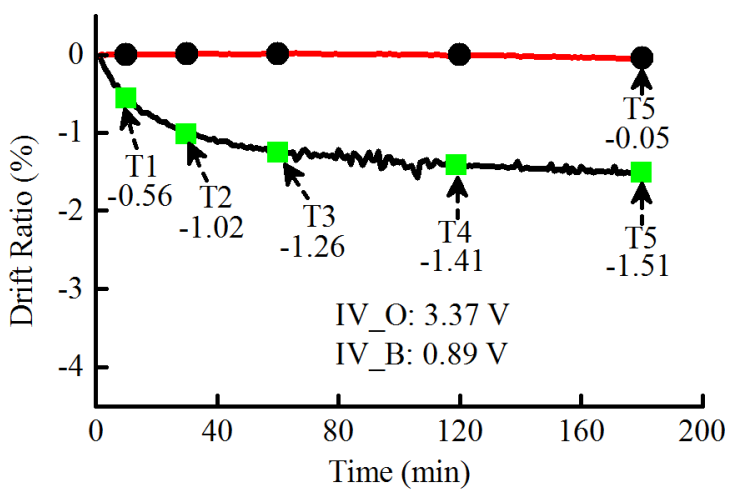

(a)

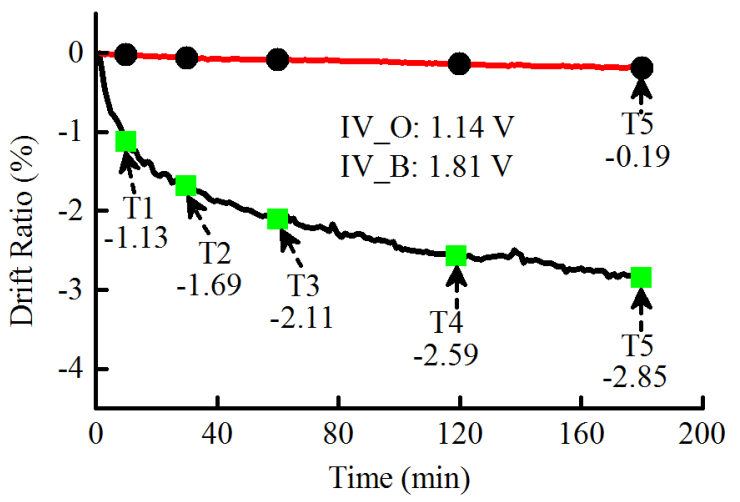

(c)

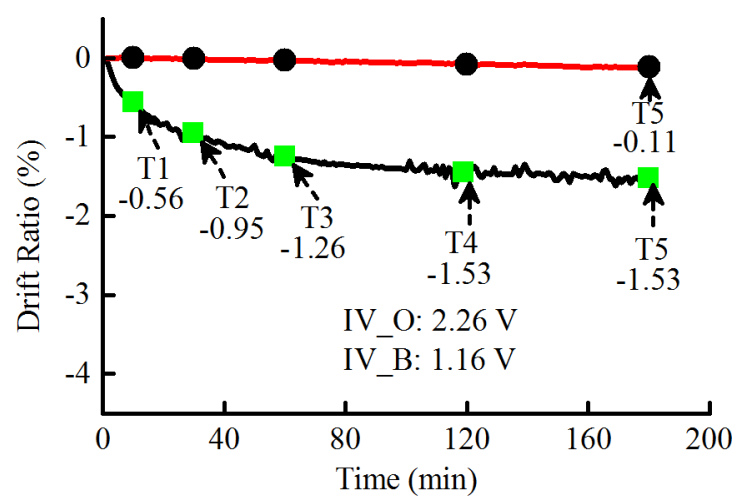

(b)

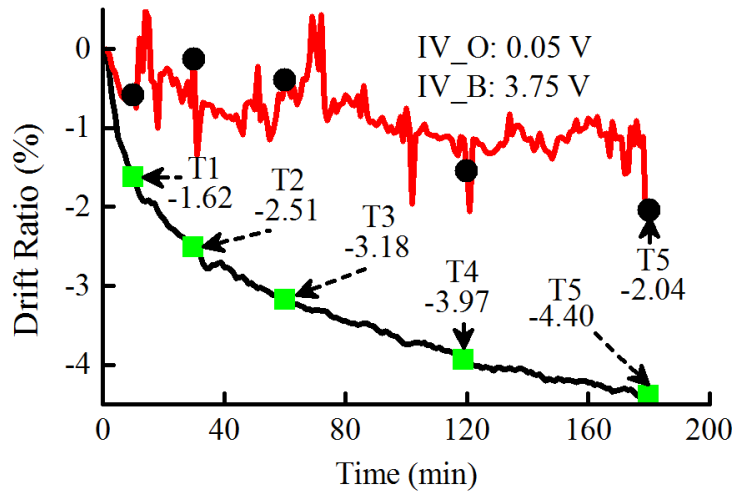

(d)

\section{-Optical sensor $\quad-$ - Bend sensor}

Fig. 9. Drift ratios of the optical sensor and the bend sensor at different bending angles over 180 minutes: (a) is the result with the flexion angle of $30^{\circ}$; (b) for $45^{\circ}$; (c) for $60^{\circ}$; and (d) for $90^{\circ}$. IV_O represents the initial voltage reading of the optical sensor at the bending position, and IV_B is for the bend sensor.

\section{Performance Under Static Conditions}

In this part, the methods for measuring the static characteristics, including nonlinearity, hysteresis, repeatability, and stability, are described for both the optical and commercial bend sensors. A separate coordinate system is defined for each sensor. For the optical sensor, the physical position where the sensor wings line up is defined as $0^{\circ}$. For the commercial bend sensor, the natural flat position is defined as $0^{\circ}$. The rotation in a clockwise direction is a move from a smaller absolute position to a larger one, and the anticlockwise rotation denotes the opposite.

The measurements are conducted under the same room temperature conditions, the motor performs rotations at $10 \%$ and data acquisition is carried out at 100 samples per second. Initialization of the system ensures that the $0^{\circ}$ position of each sensor is aligned with the zero scale of the stepper motor stage.

In the first test, the sensors are rotated (optical) or flexed (bend) through angles ranging from $-20^{\circ}$ to $100^{\circ}$ and then back to $-20^{\circ}$ with increments of $5^{\circ}$. For each angle setting, up to 500 samples are taken. This process is repeated five times with an interval of three minutes between each sampling cycle (i.e. 50 steps per cycle, 500 samples per angle step).

The other test focuses on the stability evaluation. Stability is the capacity of a sensor to remain steady under the same measurement conditions. In this test, the sensors rotate to a specific bending angle, and then remain at that position for up to 180 minutes during which data is continuously acquired.
The sensor performances are investigated at bending angles of $30^{\circ}, 45^{\circ}, 60^{\circ}$, and $90^{\circ}$, successively.

\section{Performance Under Dynamic Conditions}

The dynamic characteristics of each sensor is assessed by using the stepper motor in continuous mode. In this test, the motor is rotated at the maximum angular acceleration of 70 degrees per second squared allowed by the experimental apparatus. The sensor data is acquired at a rate of $10 \mathrm{kHz}$ in synchronization with the motor controller. Note that $\mathrm{C}_{2}$ in Fig. 6 (a) is removed to eliminate the delay introduced by the filters during this experiment.

The bend sensor shows a linear performance with a sensitivity of $57 \mathrm{mV}$ per degree above $40^{\circ}$ (see Fig. 8 and (6)) whereas the optical sensor is most sensitive for angles between $20^{\circ}$ to $70^{\circ}$ (see Fig. 7). Therefore, we monitor their responses in the range from $40^{\circ}$ to $70^{\circ}$ where both sensors possess a high signal-to-noise ratio. The measurement procedure involves increasing the angle from $40^{\circ}$ to $70^{\circ}$ and then back again to $40^{\circ}$ for five cycles. A two-second delay is included at the beginning and end of each cycle.

The motor acceleration and the test range, limit the maximum speed of the motor to $45.8 \%$ s. Therefore, we investigate the sensor response at different rotation speeds of $15 \%, 25 \%$, $35 \%$, and $45 \%$. 
TABLE I

REPEATABILITY VALUES

\begin{tabular}{lll}
\hline Sensors & Mean SR & SD \\
\hline $\begin{array}{l}\text { Optical sensor } \\
\text { (Our study) }\end{array}$ & $0.03^{\circ}$ & $0.01^{\circ}$ \\
$\begin{array}{l}\text { Bend sensor } \\
\text { (Tested in this paper) }\end{array}$ & $0.29^{\circ}$ & $0.11^{\circ}$ \\
$\begin{array}{l}\text { Fibre optic sensor } \\
\text { (DataGlove) [32] }\end{array}$ & $5.60^{\circ}$ & $2.30^{\circ}$ \\
$\begin{array}{l}\text { Hall effect sensor } \\
\text { (HumanGlove) [33] }\end{array}$ & $6.65^{\circ}$ & $2.14^{\circ}$ \\
$\begin{array}{l}\text { Optical linear encoder } \\
\text { (SmartGlove)* [34] }\end{array}$ & $3.29^{\circ}$ & $3.06^{\circ}$ \\
$\begin{array}{l}\text { Bend sensor } \\
\text { (WU Glove) [25] }\end{array}$ & $4.96^{\circ}$ & $1.59^{\circ}$ \\
$\begin{array}{l}\text { Bend sensor } \\
\text { (Shadow Monitor)* [26] }\end{array}$ & $3.36^{\circ}$ & $1.05^{\circ}$ \\
$\begin{array}{l}\text { Bend sensor } \\
\text { (HITEG-Glove)* [24] }\end{array}$ & $3.25^{\circ}$ & $1.07^{\circ}$ \\
\hline
\end{tabular}

*Note: The performance of the sensors/sensing gloves were obtained using real hands.

\section{Results AND Discussions}

\section{A. Sensor Static Performance}

In this part, we compare the static performance of both the optical and the bend sensors, and also discuss their nonlinearity, hysteresis errors, repeatability, and stability.

1) Nonlinearity: By averaging the sampled data at each angle, we obtain the output voltages in both clockwise and anticlockwise rotations. The angular dependence of the output of the optical sensor is shown in Fig. 7. According to (4), $n$ is the output span $(4.43 \mathrm{~V})$ and $b$ is the offset voltage of this sensor $(0.05 \mathrm{~V})$. The theoretical values are calculated and also plotted in Fig. 7. It can be seen that the measured output voltages of the optical sensor are consistent with the theoretical values for both directions of rotation. The degree of average deviation from the theoretical values is $1.2 \%$ during the clockwise rotation, and $2.1 \%$ during the anticlockwise rotation, so hence most measurement readings fall between $2 \%$ of the theoretical values during bidirectional rotations. Although the output voltage should be $0 \mathrm{~V}$ in theory when the rotation angle is $90^{\circ}$, an offset voltage of approximately $50 \mathrm{mV}$ exists, as shown in Fig. 7. There may be several reasons for this including polarizer defects, misalignment of the two pieces of polarizing film, and input bias current of the amplifier.

As is illustrated in Fig. 8, the commercial bend sensor is insensitive to small bending angles under $20^{\circ}$ and demonstrates a piecewise linear performance. Averaging the results in clockwise and anticlockwise directions at each angle, we fit a linear function to two angular ranges, shown as (6).

$$
V=\left\{\begin{array}{lr}
0.011 x+0.605, & 20 \leqslant x<40 \\
0.057 x-1.467, & x \geqslant 40
\end{array}\right.
$$

where $V$ is the output voltage in volts, and $x$ is the bending angle in degrees.
For the bend sensor, when the measured voltage is converted to an angle using (6), the root-mean-square error (RMSE) is $2.0^{\circ}$ (over the $20^{\circ}$ to $100^{\circ}$ measuring range) relative to the motor angular positions (accurate to $0.08^{\circ}$ ). In contrast, the optical sensor only suffers from a RMSE of $0.5^{\circ}$, much smaller than the bend sensor. In addition, the optical sensor is also more accurate than the FBG sensor (maximum error: $2.0^{\circ}$ ) [19], single-mode optical fibre sensor $\left(1.4^{\circ}\right)$ [30], the embedded hetero-core fibre-optic sensor $\left(0.9^{\circ}\right)$ [18], and the linear potentiometer (average error: $0.7^{\circ}$ ) [5].

2) Hysteresis: Under the specified testing conditions, hysteresis is more apparent in the bend sensor compared to the optical sensor. The following equation is employed to quantify the hysteresis errors [31].

$$
\delta_{H}(i)=\frac{|\Delta V(i)|}{V_{\max }-V_{\min }} \cdot 100 \%, \quad i=-20,-15, \ldots 100
$$

where $\Delta V(i)$ denotes the difference of the sensor outputs between the clockwise and anticlockwise readings at the given bending angle $i . V_{\max }$ and $V_{\min }$ are the maximum and minimum output voltages in the testing range from $-20^{\circ}$ to $100^{\circ}$, respectively.

The calculation results show that the hysteresis of the optical sensor is less than $1.07 \%$, with an average of $0.55 \%$, while the bend sensor possesses a hysteresis up to $2.35 \%$ (average is $1.02 \%$ ). Thus the performance of the optical sensor is normally less dependent on the history state than the bend sensor.

3) Repeatability: The Statistical Range (SR) and standard deviation (SD) have been previously used to assess the repeatability of sensors used in glove-based systems [24]-[26], [32]-[34]. Here, we use the same numerical methods to quantify the sensor repeatability but under more controlled testing conditions compared to those performed on hand models. At each testing position, 500 voltage measurements are averaged, and then converted to angles using the voltage-to-angle relationship. The difference between the highest and lowest angles measured over five cycles is computed for each testing angle. The mean SR, as well as the overall SD, are listed in TABLE I for both the optical and bend sensors. The repeatability of the bend sensor is only investigated over the $20^{\circ}$ to $100^{\circ}$ range since the output is undetectable below $20^{\circ}$. TABLE I also includes performance data of sensors reported by other groups. These sensors were integrated into gloves or supports, and the repeatability was investigated by using real hand models. This is likely to produce exaggerated repeatability values compared to the ideal conditions used in our measurements.

From TABLE I, it can be observed that the designed optical sensor has significantly better repeatability than the bend sensor. Other sensors, including fibre optic, Hall Effect, and optical linear encoders, show higher SR and SD but these are obtained under less controlled conditions, i.e. mounted on real hands. The HITEG-Glove, Shadow Monitor, and WU glove, adopting bend sensors from Flexpoint Inc., are more repeatable than the Hall Effect and Fibre optic based glove systems. Our optical sensor, with much better repeatability albeit under ideal conditions, shows great promise as a sensory element for future glove-based systems. 


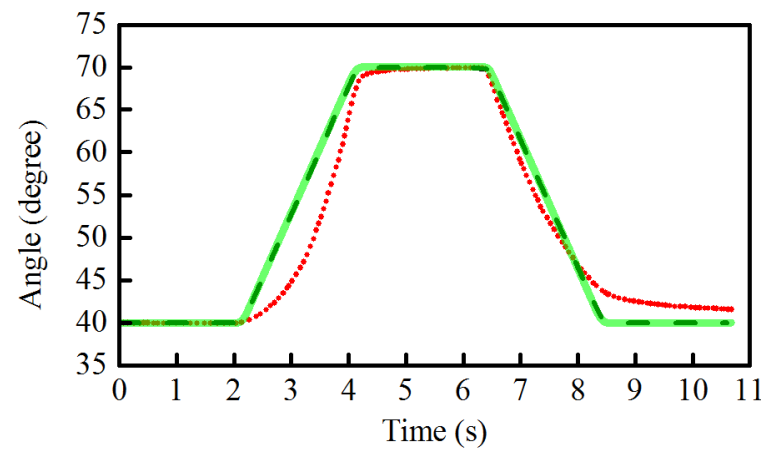

(a) At the maximum velocity of $15 \%$

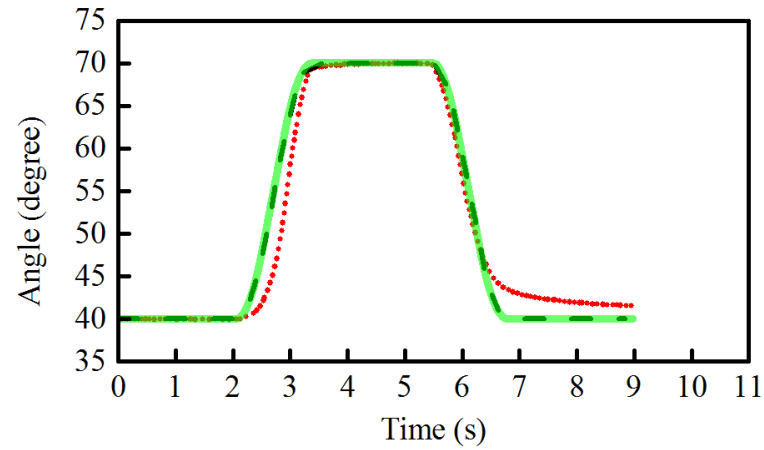

(c) At the maximum velocity of $35 \% \mathrm{~s}$

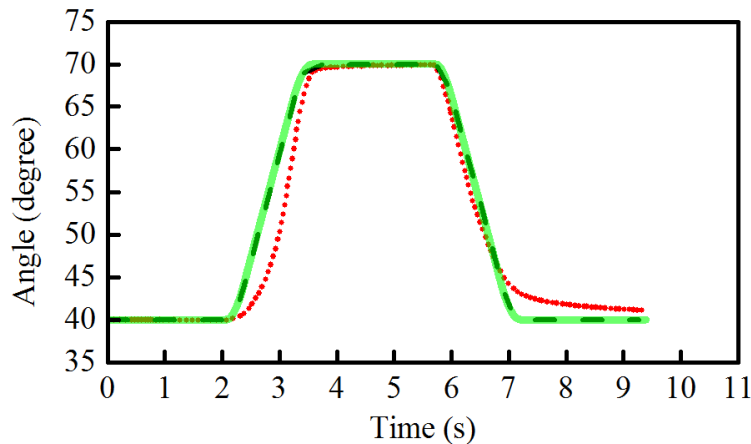

(b) At the maximum velocity of $25 \% \mathrm{~s}$

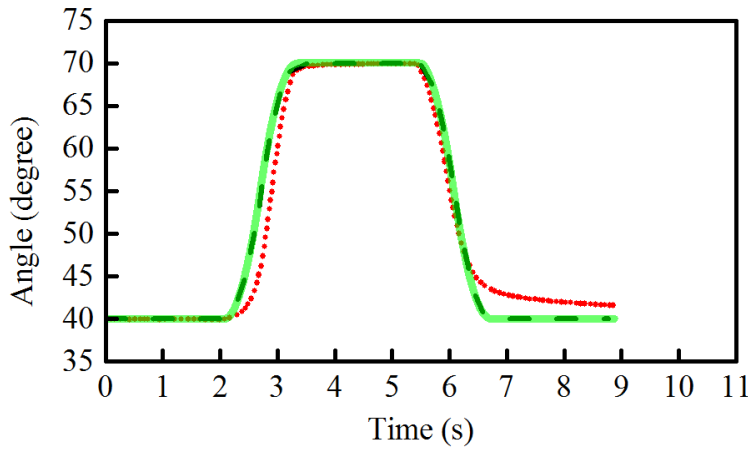

(d) At the maximum velocity of $45 \% \mathrm{~s}$

$$
\text { - - Motor }=\text { Optical sensor } \cdots \cdots \cdot \text {.... Bend sensor }
$$

Fig. 10. Dynamic performance of the developed optical sensor and the bend sensor at different rotation speeds: (a) is the sensor performance at the velocity of $15 \%$; (b) for $25 \%$; (c) for $35 \%$; and (d) for $45 \%$ s.

4) Stability: Sensor stability was assessed by specifying a fixed bending angle and measuring any change in output over an extended period of time. The relative change in output, i.e. drift ratio, was measured for both types of sensor at bending positions of $30^{\circ}, 45^{\circ}, 60^{\circ}$, and $90^{\circ}$. At each bending state, the values of percentage drift after $10 \mathrm{~min}, 30 \mathrm{~min}, 60 \mathrm{~min}$, $120 \mathrm{~min}$, and $180 \mathrm{~min}$ (T1-T5) have been superimposed on the drift curve in Fig. 9. The results in Fig. 9 show that the optical sensor has significantly less drift compared to the bend sensor over the maximum measurement period of 180 minutes. In the case of the bend sensor, there is a clear increase in signal drift with bend angle, i.e. $1.5 \%$ to $4.4 \%$ with bending from $30^{\circ}$ to $90^{\circ}$. In contrast, the optical sensor's drift values are much smaller; the absolute values close to constant irrespective of the bending angle. The larger drift ratio at $90^{\circ}$ is a consequence of the very low output signal amplitude compared to the absolute drift value for this measurement. The increased drift in the bend sensor is however indicative of some form of structural relaxation and increases significantly at larger bend angles.

\section{B. Sensor Dynamic Response}

The rotation stage moves after receiving a command from the motor controller and then returns a confirmatory message back to the controller. Time delays associated with this communication were not provided by the manufacturer. Therefore, in this work it was reasonable to assume that the communication time delays were small in comparison to the rotation speeds used to simulate joint movement.
In the testing procedure, the motor rests for 2 seconds at $40^{\circ}$, followed by a rapid rotation to $70^{\circ}$, and this is maintained for a further 2 seconds followed by a reverse rotation back to $40^{\circ}$. Fig. 10 shows the dynamic response of both the optical and the bend sensors. The optical sensor exhibits identical behaviour to that of the motor effectively providing an instantaneous response relative to the speeds investigated here $\left(15,25,35,45^{\circ} / \mathrm{s}\right)$. In contrast, the bend sensor exhibits an initial time delay (increasing to $0.6 \mathrm{~s}$ at $15 \%$ ) followed by a complicated response consisting of a negative to positive time delay transition. There is clearly a problem with mechanical hysteresis with the bend sensor where the final reading does not return to the starting value.

It can be declared that the optical sensor possesses a better dynamic response than the commercial bend sensor under the test velocities investigated here.

\section{Conclusions}

The excellent performance of the 3D printed optical sensor reveals good potential as a sensory element for monitoring hand posture and movement. Compared with a commercial bend sensor, the optical sensor demonstrates superior characteristics in terms of accuracy, repeatability, stability, as well as its dynamic response. The designed optical sensor is also more accurate and repeatable in comparison with other sensors reported by several other groups. The compact hinged configuration enables the optical sensor to track the physical bending angles directly without obstructing or restricting normal hand movements. Time-consuming calibration procedures are also 
not required making it ideal for integration into a glovebased system for tracking hand movements. The optical sensor is suitable for attaching to the dorsal surface of the finger, via a glove, to track flexion or extension. This is consistent with standard practice using clinical goniometers. The optical sensor is designed to operate in the range of $0^{\circ}$ to $90^{\circ}$. This measuring range is sufficient for most finger activities, although this will need extending up to $120^{\circ}$ if the full range of hand movement is to be achieved. Furthermore, the sensor could be adapted to monitor other parts of body, e.g. the knee, wrist or any other articulating joint. The sensor is restricted to a single axis of rotation and therefore cannot be used to monitor abduction/adduction. A multi-axis sensor is currently being developed to overcome this issue and will reported in a later publication.

The optical sensor has demonstrated good performance under laboratory test conditions. The performance under realworld conditions will depend on many other factors, the most important being the method of mechanical coupling to the hand. Future work will include extending the angular range and degrees of freedom of measurement and test the performance on real hand models.

\section{REFERENCES}

[1] L. Dipietro, A. M. Sabatini, and P. Dario, "A survey of glove-based systems and their applications," IEEE Trans. Syst., Man, Cybern. C, Appl. Rev., vol. 38, no. 4, pp. 461-482, Jul. 2008.

[2] D. J. Sturman and D. Zeltzer, "A survey of glove-based input," IEEE Comput. Graph. Appl., vol. 14, no. 1, pp. 30-39, Jan. 1994.

[3] G. S. Rash, P. P. Belliappa, M. P. Wachowiak, N. N. Somia, and A. Gupta, "A demonstration of the validity of a 3-D video motion analysis method for measuring finger flexion and extension," J. Biomech., vol. 32, no. 12, pp. 1337-1341, Dec. 1999.

[4] J. Park and Y.-L. Yoon, "LED-glove based interactions in multi-modal displays for teleconferencing," in Proc. ICAT, Hangzhou, Nov. 2006, pp. 395-399.

[5] Y. Park, J. Lee, and J. Bae, "Development of a wearable sensing glove for measuring the motion of fingers using linear potentiometers and flexible wires," IEEE Trans. Ind. Informat., vol. 11, no. 1, pp. 198-206, Feb. 2015

[6] G. Saggio, "Mechanical model of flex sensors used to sense finger movements," Sens. Actuators A, Phys., vol. 185, pp. 53-58, Oct. 2012.

[7] Y. Wang et al., "Wearable and highly sensitive graphene strain sensors for human motion monitoring," Adv. Funct. Mater., vol. 24, pp. 4666-4670, Apr. 2014.

[8] M. Amjadi, A. Pichitpajongkit, S. Lee, S. Ryu, and I. Park, "Highly stretchable and sensitive strain sensor based on silver nanowireelastomer nanocomposite," ACS Nano, vol. 8, no. 5, pp. 5154-5163, 2014.

[9] R. K. Kramer, C. Majidi, R. Sahai, and R. J. Wood, "Soft curvature sensors for joint angle proprioception," in Proc. IROS, San Francisco, CA, USA, Sep. 2011, pp. 1919-1926.

[10] T. Yamada et al., "A stretchable carbon nanotube strain sensor for human-motion detection," Nature Nanotechnol., vol. 6, pp. 296-301, Mar. 2011.

[11] N. W. Williams, J. M. T. Penrose, C. M. Caddy, E. Barnes, D. R. Hose, and P. Harley, "A goniometric glove for clinical hand assessment construction, calibration and validation," J. Hand Surg., vol. 25, no. 2, pp. 200-207, Apr. 2000.

[12] C.-S. Fahn and H. Sun, "Development of a data glove with reducing sensors based on magnetic induction," IEEE Trans. Ind. Electron., vol. 52, no. 2, pp. 585-594, Apr. 2005.

[13] K.-Y. Chen, K. Lyons, S. White, and S. Patel, "uTrack: 3D input using two magnetic sensors," in Proc. UIST, New York, NY, USA, Oct. 2013 , pp. 237-244.

[14] Y. Ma, Z.-H. Mao, W. Jia, C. Li, J. Yang, and M. Sun, "Magnetic hand tracking for human-computer interface," IEEE Trans. Magn., vol. 47, no. 5, pp. 970-973, May 2011.
[15] H. G. Kortier, J. Antonsson, H. M. Schepers, F. Gustafsson, and P. H. Veltink, "Hand pose estimation by fusion of inertial and magnetic sensing aided by a permanent magnet," IEEE Trans. Neural Syst. Rehabil. Eng., vol. 23, no. 5, pp. 796-806, Sep. 2015.

[16] A. Babchenko and J. Maryles, "A sensing element based on 3D imperfected polymer optical fibre," J. Opt. A, Pure Appl. Opt., vol. 9, no. 1, pp. 1-5, Nov. 2006.

[17] L. Bilro, J. G. Oliveira, J. L. Pinto, and R. N. Nogueira, "A reliable lowcost wireless and wearable gait monitoring system based on a plastic optical fibre sensor," Meas. Sci. Technol., vol. 22, no. 4, p. 045801, Mar. 2011.

[18] M. Nishiyama and K. Watanabe, "Wearable sensing glove with embedded hetero-core fiber-optic nerves for unconstrained hand motion capture," IEEE Trans. Instrum. Meas., vol. 58, no. 12, pp. 3995-4000, Dec. 2009.

[19] A. F. da Silva, A. F. Goncalves, P. M. Mendes, and J. H. Correia, "FBG sensing glove for monitoring hand posture," IEEE Sensors J., vol. 11 , no. 10 , pp. $2442-2448$, Oct. 2011.

[20] E. Fujiwara, M. F. M. D. Santos, and C. K. Suzuki, "Flexible optical fiber bending transducer for application in glove-based sensors," IEEE Sensors J., vol. 14, no. 10, pp. 3631-3636, Oct. 2014.

[21] B. Lee, "Review of the present status of optical fiber sensors," Opt. Fiber Technol., vol. 9, no. 2, pp. 57-79, 2003.

[22] M. Nishiyama, H. Sasaki, and K. Watanabe, "Optical intensity-based measurement of multipoint hetero-core fiber sensors by the method of time-differentiation in optical loss," IEEE Sensors J., vol. 8, no. 7, pp. 1055-1060, Jul. 2008.

[23] (2015). Flexpoint Sensor Systems, Inc. [Online]. Available: http://www. flexpoint.com/

[24] G. Saggio, "A novel array of flex sensors for a goniometric glove," Sens. Actuators A, Phys., vol. 205, pp. 119-125, Jan. 2014.

[25] R. Gentner and J. Classen, "Development and evaluation of a low-cost sensor glove for assessment of human finger movements in neurophysiological settings," J. Neurosci. Methods, vol. 178, no. 1, pp. 138-147, Mar. 2009.

[26] L. K. Simone, N. Sundarrajan, X. Luo, Y. Jia, and D. G. Kamper, "A low cost instrumented glove for extended monitoring and functional hand assessment," J. Neurosci. Methods, vol. 160, no. 2, pp. 335-348, Mar. 2007.

[27] L. Wang, T. Meydan, P. Williams, and K. T. Wolfson, "A proposed optical-based sensor for assessment of hand movement," in Proc. IEEE SENSORS, Busan, South Korea, Nov. 2015, pp. 1-4.

[28] G. Saggio, F. Riillo, L. Sbernini, and L. R. Quitadamo, "Resistive flex sensors: A survey," Smart Mater. Struct., vol. 25, no. 1, p. 3001, Dec. 2015.

[29] (2016). Thorlabs, Inc. [Online]. Available: http://www.thorlabs.co.uk/ newgrouppage 9. cfm?objectgroupid $=1064 \& \mathrm{pn}=\mathrm{NR} 360 \mathrm{~S} / \mathrm{M} \# 2605$

[30] M. Donno, E. Palange, F. D. Nicola, G. Bucci, and F. Ciancetta, "A new flexible optical fiber goniometer for dynamic angular measurements: Application to human joint movement monitoring," IEEE Trans. Instrum. Meas., vol. 57, no. 8, pp. 1614-1620, Aug. 2008.

[31] J. G. D. Silva, A. A. D. Carvalho, and D. D. D. Silva, "A strain gauge tactile sensor for finger-mounted applications," IEEE Trans. Instrum. Meas., vol. 51, no. 1, pp. 18-22, Feb. 2002.

[32] S. Wise et al., "Evaluation of a fiber optic glove for semi-automated goniometric measurements," J. Rehabil. Res. Develop., vol. 27, no. 4, pp. 411-424, 1990.

[33] L. Dipietro, A. M. Sabatini, and P. Dario, "Evaluation of an instrumented glove for hand-movement acquisition," J. Rehabil. Res. Develop., vol. 40, no. 2, pp. 179-190, Mar./Apr., 2003.

[34] K. Li, I.-M. Chen, S. H. Yeo, and C. K. Lim, "Development of fingermotion capturing device based on optical linear encoder," J. Rehabil. Res. Develop., vol. 48, no. 1, pp. 69-82, 2011.

Lefan Wang received the M.Eng. degree in electrical and electronic enginnering from the Harbin Institute of Technology, China. She is currently pursuing the Ph.D. degree with the Wolfson Centre for Magnetics, School of Engineering, Cardiff University. Her Ph.D. degree is sponsored by the China Scholarship Council and Cardiff University.

Turgut Meydan (M'88) is currently a Reader with the Wolfson Centre for Magnetics, School of Engineering, Cardiff University, U.K.

Paul Williams is currently a Research Fellow with the Wolfson Centre for Magnetics, School of Engineering, Cardiff University, U.K. 